\title{
Effects of category learning on the stimulus selectivity of macaque inferior temporal neurons
}

\author{
Wouter De Baene, ${ }^{1}$ Bart Ons, ${ }^{2}$ Johan Wagemans, ${ }^{2}$ and Rufin Vogels ${ }^{1,3}$ \\ ${ }^{1}$ Laboratorium voor Neuro- en Psychofysiologie, K.U. Leuven Medical School, Campus Gasthuisberg, Leuven B-3000, Belgium; \\ ${ }^{2}$ Laboratory of Experimental Psychology, K.U. Leuven, Leuven B-3000, Belgium
}

\begin{abstract}
Primates can learn to categorize complex shapes, but as yet it is unclear how this categorization learning affects the representation of shape in visual cortex. Previous studies that have examined the effect of categorization learning on shape representation in the macaque inferior temporal (IT) cortex have produced diverse and conflicting results that are difficult to interpret owing to inadequacies in design. The present study overcomes these issues by recording IT responses before and after categorization learning. We used parameterized shapes that varied along two shape dimensions. Monkeys were extensively trained to categorize the shapes along one of the two dimensions. Unlike previous studies, our paradigm counterbalanced the relevant categorization dimension across animals. We found that categorization learning increased selectivity specifically for the category-relevant stimulus dimension (i.e., an expanded representation of the trained dimension), and that the ratio of within-category response similarities to between-category response similarities increased for the relevant dimension (i.e., category tuning). These small effects were only evident when the learned category-related effects were disentangled from the prelearned stimulus selectivity. These results suggest that shape-categorization learning can induce minor category-related changes in the shape tuning of IT neurons in adults, suggesting that learned, category-related changes in neuronal response mainly occur downstream from IT.
\end{abstract}

Lesion (Dean 1976), microstimulation (Afraz et al. 2006), and single-cell studies (Gross et al. 1969; Logothetis and Sheinberg 1996; Tanaka 1996) indicate that the inferior temporal (IT) cortex is involved in the processing of features necessary for the identification and categorization of objects. Single-cell studies of category encoding by IT neurons (Vogels 1999b; Sigala and Logothetis 2002; Freedman et al. 2003; Kiani et al. 2007) reported that, while some single IT neurons responded selectively to exemplars of a particular category, these single IT neurons still showed selectivity for different exemplars within that category. The output of a population of IT neurons can be used to classify exemplars of different categories (Thomas et al. 2001; Kayaert et al. 2005; Kiani et al. 2007), suggesting a population coding of features that is biased toward category-relevant distinctions between stimuli. Here we address the question of whether this sort of categoryrelated bias in IT neuronal sensitivity can be induced by categorization learning. Indeed, the tuning of IT neurons could be susceptible to categorization learning, as neuronal responses in the IT cortex can reflect the perceptual similarity across stimuli (Op de Beeck et al. 2001; Kayaert et al. 2003) and the latter can be affected by categorization (Goldstone 1994; Livingston et al. 1998; Goldstone et al. 2001).

Previous studies of the effect of categorization learning on the tunings of IT neurons have obtained diverse results. Sigala and Logothetis (2002) reported enhanced selectivity for shape features that were relevant (compared with irrelevant) for the categorization. However, the relatively large effect of categorization-relevant feature selectivity could merely be due to stimulus selectivity, unrelated to categorization learning, since no pretraining selectivity measurements were obtained and the diagnostic features were identical in both monkeys. Freedman et al. (2003) found that responses to shapes within a learned category were slightly more similar than responses to shapes from differ-

${ }^{3}$ Corresponding author.

E-mail Rufin.Vogels@med.kuleuven.be; fax 32-16-330027.

Article is online at http://www.learnmem.org/cgi/doi/10.1101/lm.1040508. ent categories. However, this small effect could also have resulted from physical differences amongst the stimuli rather than differences in category membership. Finally, Op de Beeck et al. (2001) found no effect of a learned categorization rule upon the selectivity's of IT neurons. A possible explanation for the discrepancy between the Op de Beeck et al. (2001) and Sigala and Logothetis (2002) studies could be that Sigala and Logothetis manipulated separable dimensions, while Op de Beeck et al. used integral dimensions. Separable dimensions, but not integral dimensions, can be attended to separately (Garner 1974). Indeed, a human psychophysical study (Op de Beeck et al. 2003) found that categorization learning induced a dimension-specific gain in perceptual similarity for shapes that varied along separable, but not integral, dimensions. Given these discrepant results, we revisited the effect of categorization learning on IT shape selectivity. We controlled for prelearned stimulus-selectivity effects by measuring the selectivity's of IT neurons both before and after learning and by counterbalancing the relevant categorization dimensions across animals. We manipulated shape dimensions that had previously been shown to be separable in human psychophysical studies (e.g., Op de Beeck et al. 2003; Wagemans et al. 2006).

\section{Results}

Before starting the categorization training, we measured pretraining "baseline" IT responses to four different parametric sets of 16 shapes each. In each set, the 16 shapes differed along a "curvature" and "aspect ratio" dimension (Fig. 1). Responses were measured using a rapid serial visual presentation (RSVP) paradigm while the monkeys were passively fixating. In a subsequent categorization-training phase, one monkey was trained to group stimuli into two categories based on the curvature dimension (monkey C; training duration: $53 \mathrm{~d}$ ); the other monkey was trained to use the aspect ratio dimension (monkey A; training duration: $80 \mathrm{~d}$ ) to categorize the shapes. After this training phase, we recorded IT responses while the monkeys were categorizing the shapes (post-training recordings). 


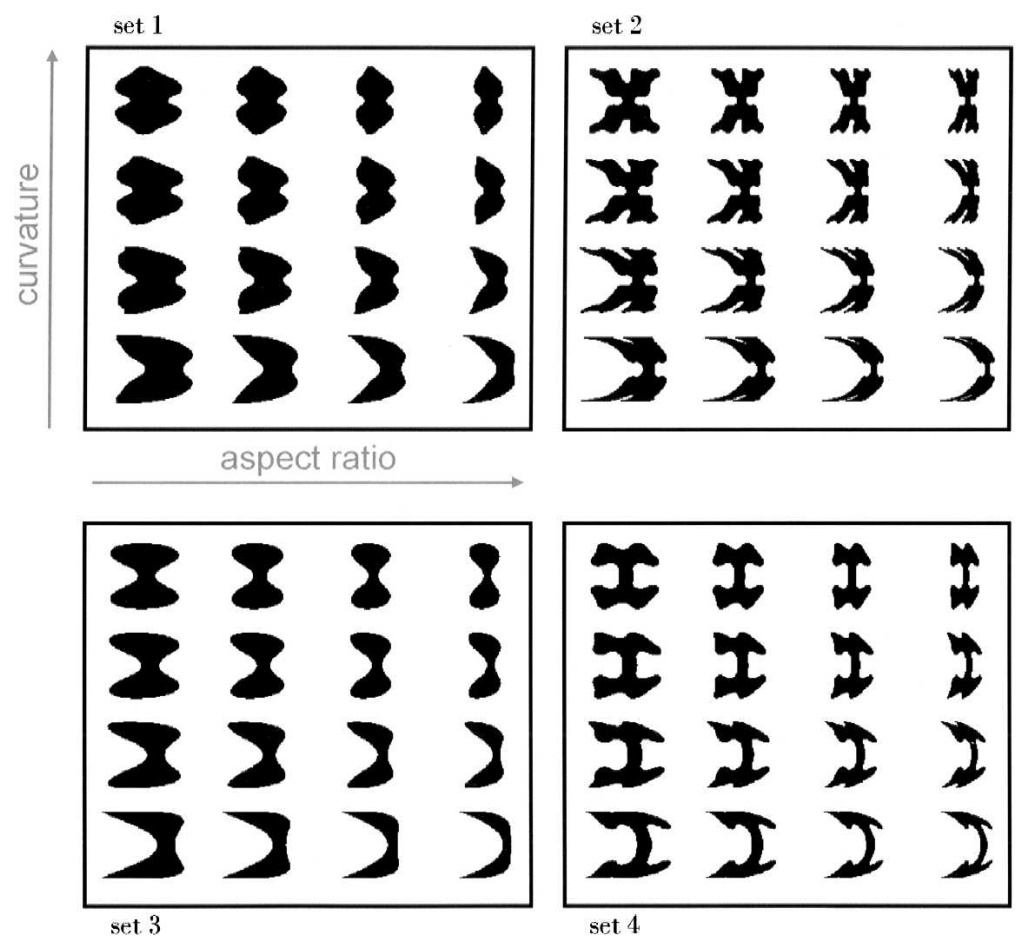

Figure 1. Stimuli. The square parametric configurations consisted of four sets of 16 shapes each. The manipulated dimensions were curvature and aspect ratio (vertical and horizontal axis, respectively). Curvature was modulated by manipulating the turning angle of the tangents at the shape ends on the vertical axis. Aspect ratio was modulated by manipulating shape width.

\section{Behavioral results}

The behavioral results of the two monkeys during the last $10 \mathrm{~d}$ of training (Fig. 2) indicate that both animals were fairly adept at categorizing the shapes that were used in the post-training recordings. While shapes close to the extreme parametric values ( $0 \%$ or $100 \%)$ were categorized almost perfectly, these subjects reached a performance level of about $85 \%$ correct for shapes with moderate parametric values (around 33\% and 66\%).

Averaged over all post-training recording sessions, monkey $\mathrm{C}$, who used curvature as the relevant dimension, reached a performance of $98 \%$ in categorizing the 16 shapes of Figure 1. For the position- and size-control shapes (see Materials and Methods), categorization was correct in $99 \%$ and $97 \%$ of the trials, respectively. Monkey $\mathrm{A}$, who used aspect ratio as the relevant dimension, categorized the 16 shapes correctly on $97 \%$ of the trials. He reached a performance of $96 \%$ for the position control shapes and $95 \%$ for the size control shapes. Thus, both animals performed with a high accuracy in the categorization task during the recording sessions.

\section{Neuronal results}

\section{Database}

Pretraining recording phase

We recorded from 214 neurons (109 cells in monkey C; 105 cells in monkey A), 201 of which showed significant shape selectivity within one or more shape sets (101 cells in monkey C; 100 cells in monkey A). This resulted in a significant response modulation for 479 nonrotated shape sets, as tested with a permutation test (see Materials and Methods). For each cell, there was an average of 98.66 presentations per stimulus (minimum, 36; maximum, 152).

\section{Post-training recording phase}

We recorded from 137 neurons (70 cells in monkey C, 67 cells in monkey A) using the same stimulus centering as in the pretraining recording phase (center type 1 ; see Materials and Methods). All of these neurons were responsive to at least one set, as tested with a split-plot design analysis of variance (ANOVA) (Kirk 1995). These 137 responsive cells provided us with 283 sets, for which a significant response occurred (further denoted as responsive sets). For 215 of these 283 sets, there was significant shape selectivity (further denoted as selective sets). We recorded an additional 100 responsive neurons (48 and 52 cells in monkey $\mathrm{C}$ and monkey $\mathrm{A}$, respectively) using stimuli positioned according to center type 2 (see Materials and Methods). This resulted in 206 responsive sets, 123 of which were shape selective. Additionally, we recorded from 44 responsive neurons using center type 2 in the left hemisphere of monkey A, resulting in 74 selective sets of a total of 94 responsive sets.

As all further analyses were performed on trials with a correct response, only sets with at least one (for the computation of the Depth of Selectivity [DOS]) or two (for category tuning indices) correct trials for each stimulus were included. As a consequence, two responsive sets (both selective; center type 1) were excluded from further analyses. This resulted in an average of 8.84 correct trials per stimulus per cell (minimum, 4; maximum, 15).

\section{Comparison of pre- and post-training}

When comparing pre- and post-training data, we only included neurons in the analysis that showed a significant shape selectivity for stimulus sets recorded using the same centering (type 1) in the two training phases.

\section{Effect of categorization learning on selectivity along relevant and irrelevant dimensions}

To examine whether categorization training affected the degree of selectivity for the relevant and irrelevant dimensions, we computed the Depth Of Selectivity (DOS) index using the mean responses for the four values of a dimension, irrespective of the four values of the other dimension; the larger the DOS, the greater the selectivity. We performed an ANOVA on the DOS values for the pre- and post-training phase. The variables shape set (sets 1 to 4 ), recording phase (before or after categorization training), and monkey (monkey $\mathrm{C}$ or $\mathrm{A}$ ) were included as between-neurons variables. Category dimension (aspect ratio or curvature) was included as a within-neuron variable. The main effect of recording phase was significant $\left(F_{(1,676)}=86.40\right.$, $P<0.001)$. More interestingly, the three-way interaction between category dimension, monkey, and recording phase was significant $\left(F_{(1,676)}=13.86, P<0.001\right)$. The four-way interaction between category dimension, monkey, recording phase, and shape set was not significant $\left(F_{(3,676)}=1.34, P>0.25\right)$. Figure 3 A shows that categorization training resulted in a selectivity increase for both dimensions, but this increase was larger for the relevant dimension than for the irrelevant dimension, and this observa- 


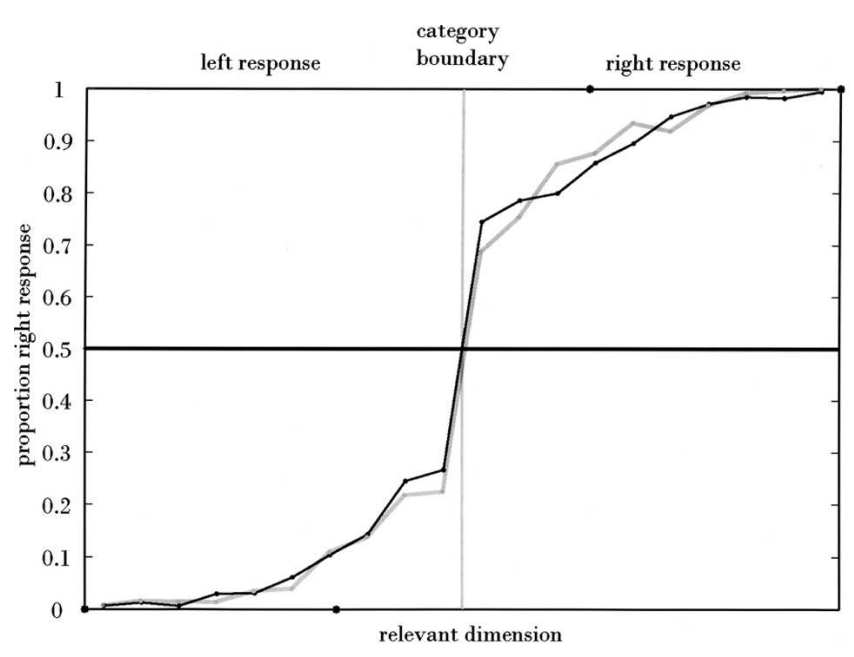

Figure 2. Behavioral performance during the last $10 \mathrm{~d}$ of the categorization-training phase. The $X$-axis indicates the parametric values along the relevant dimension, with the black markers on the $0 \%, 33 \%, 66 \%$, and $100 \%$ values. The $Y$-axis represents the average proportion of rightward saccades. The gray solid line in the middle shows the category boundary. Shapes on the left of this boundary were associated with a leftward saccade; shapes on the right with a rightward saccade. The gray and black curves show the mean performance of monkey $\mathrm{C}$ and monkey A, respectively, averaged in 20 consecutive bins, for the shapes with random parametric values used during the last $10 \mathrm{~d}$ of training.

tion held for both monkeys. Thus, categorization enhanced the selectivity more for the relevant dimension than for the irrelevant dimension (Fig. 3B,C). Indeed, the percent of neurons with a greater DOS for the relevant compared with the irrelevant dimension was larger after (54\%) compared with before training (33\%; difference: $P<0.001$, Binomial test; Fig. 3C). Highly similar, statistically significant results were found when the bestworst selectivity index employed by Sigala and Logothetis (2002) was used instead of the DOS index. The latter selectivity index was defined as the difference between the maximum and the minimum response for a given dimension divided by their sum. Furthermore, similar statistical significant effects were obtained using a best-worst index normalized by trial-to-trial variability. For this, the differences between the maximum response (averaged across presentations of the same stimulus) for a set and the minimum mean response for the same value along the relevant or irrelevant dimension were computed, followed by a division by the mean trial-to-trial standard deviation of the responses for the best and worst conditions.

\section{Effect of categorization learning on strength of category tuning}

The above analyses determined whether categorization learning induced categorization-dimension-specific changes in selectivity. Another possible categorization learning-induced change in selectivity is related to the difference in responses to shapes belonging to the same versus different learned categories. Do neurons respond more similarly to shapes that belong to the same category than to shapes that belong to different categories? To examine this, we computed a category-tuning index (see Materials and Methods) for each neuron. The category-tuning index assesses the similarity between responses to shapes of the same category versus shapes of different categories, taking into account trial-to-trial variations in response strength (computed on $\mathrm{d}^{\prime}$; see Materials and Methods). The larger the category tuning index, the more similar are the responses of neurons to shapes of the same category compared with shapes belonging to different categories. We used an ANOVA on the category tuning index values in the pre- and post-training phase. As between-neurons variables, we included shape set (set 1 to 4 ), recording phase (before or after categorization training), and monkey (monkey C or A). Category dimension (aspect ratio or curvature) was included as a within-neuron variable. The three-way interaction between category dimension, monkey, and recording phase reached significance $\left(F_{(1,673)}=3.89, P<0.05\right)$. The four-way interaction between category dimension, monkey, recording phase, and shape set was not significant $\left(F_{(3,673)}=1.84, P>0.13\right)$. Inspection of Figure 4A showed that the category-tuning index increased with training for the relevant but not for the irrelevant dimension. This observation was confirmed by post-hoc comparisons (Fisher LSD tests): For monkey C, the training effect was significant for the category-tuning index along the relevant curvature dimension $(P<0.05)$. For the category tuning index along the aspect ratio dimension, however, there was no significant training effect $(P>0.11)$. The opposite was true for monkey A $(P<0.001$ and $P>0.89$ for the training effect along the aspect ratio and curvature dimension, respectively). Thus, categorization learning affected the category tuning along the relevant dimension, but not along the irrelevant dimension (Fig. 4B,C). The distribution of the category-tuning index also changed significantly with training for the relevant but not for the irrelevant dimension $(P<0.01$ and $P>0.18$, respectively, KolmogorovSmirnov test; Fig. 4C). Similar trends were found when the analyses were applied using absolute response differences without normalizing by the response variance as Freedman et al. (2003) did, instead of using $\mathrm{d}^{\prime}$ values.

As the category-tuning index reflects the ratio of withincategory response differences (WCD's) to between-category response differences (BCD's), we zeroed in on the effect of training on both of these factors. To examine this, we used an ANOVA on the between- and within-category differences in the pre-and post-training phase, taking into account trial-to-trial variations in response strength (computed on $\mathrm{d}^{\prime}$ ). As between-neurons variables, we included shape set (set 1 to 4 ), recording phase (before or after categorization training), and monkey (monkey $\mathrm{C}$ or $\mathrm{A}$ ). Category dimension (relevant or irrelevant) and category difference (between or within) were included as within-neuron variables. The three-way interaction between category dimension, category difference, and recording phase was significant $\left(F_{(1,673)}=7.34, P<0.01\right)$. The training increased the betweencategory differences more for the relevant (post-pre training difference in mean between-category $d^{\prime}: 0.62$ ) than for the irrelevant dimension ( $\mathrm{d}^{\prime}$ difference: 0.57 ). For the within-category differences, the opposite was true: the increase for the relevant dimension ( $\mathrm{d}^{\prime}$ difference: 0.53 ) was smaller than for the irrelevant dimension ( $\mathrm{d}^{\prime}$ difference: 0.56 ). Thus, with training, responses to shapes from the same category became more similar and responses to shapes from different categories became less similar.

\section{Comparison of responses for the relevant and irrelevant dimension after training}

We also analyzed the post-training data separately, asking whether there was an interaction of stimulus dimension and monkey on the degree of selectivity and the category tuning index. These analyses are similar to those in previous studies that did not use pre-training measures. The results reported below are for the selective sets only. Including all responsive sets did not change the results in any substantial way.

\section{Selectivity along the relevant and irrelevant dimensions after training}

To examine whether selectivity for the relevant dimension was enhanced compared with the irrelevant dimension in the posttraining recording phase, we performed an ANOVA on the DOS 
A

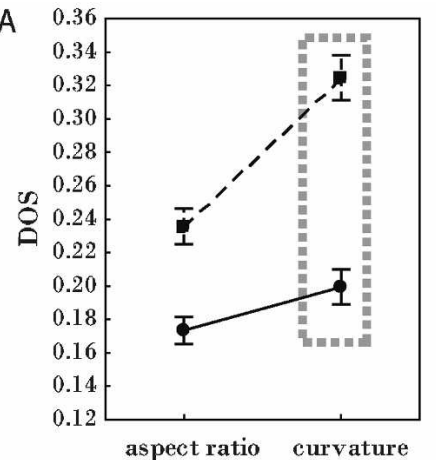

monkey $\mathrm{C}$

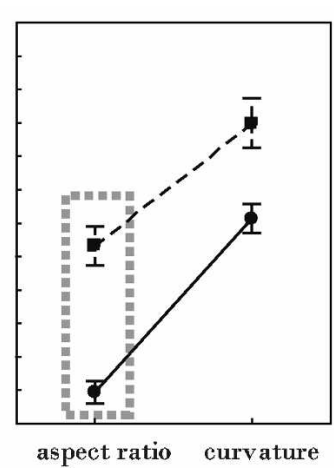

monkey A
B

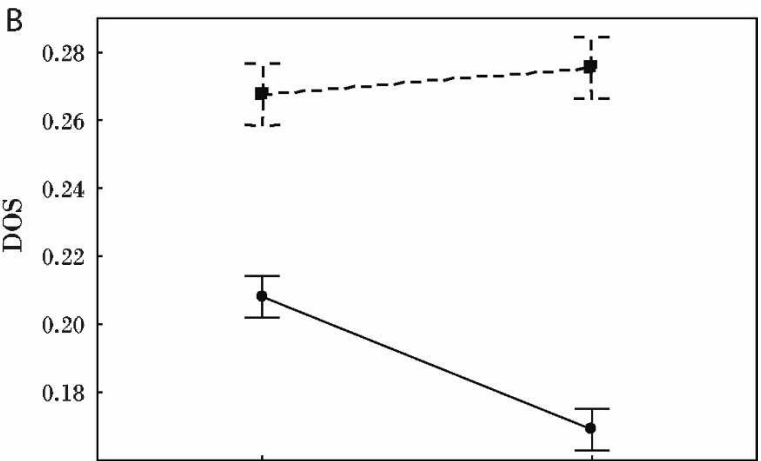

irrelevant

relevant

C
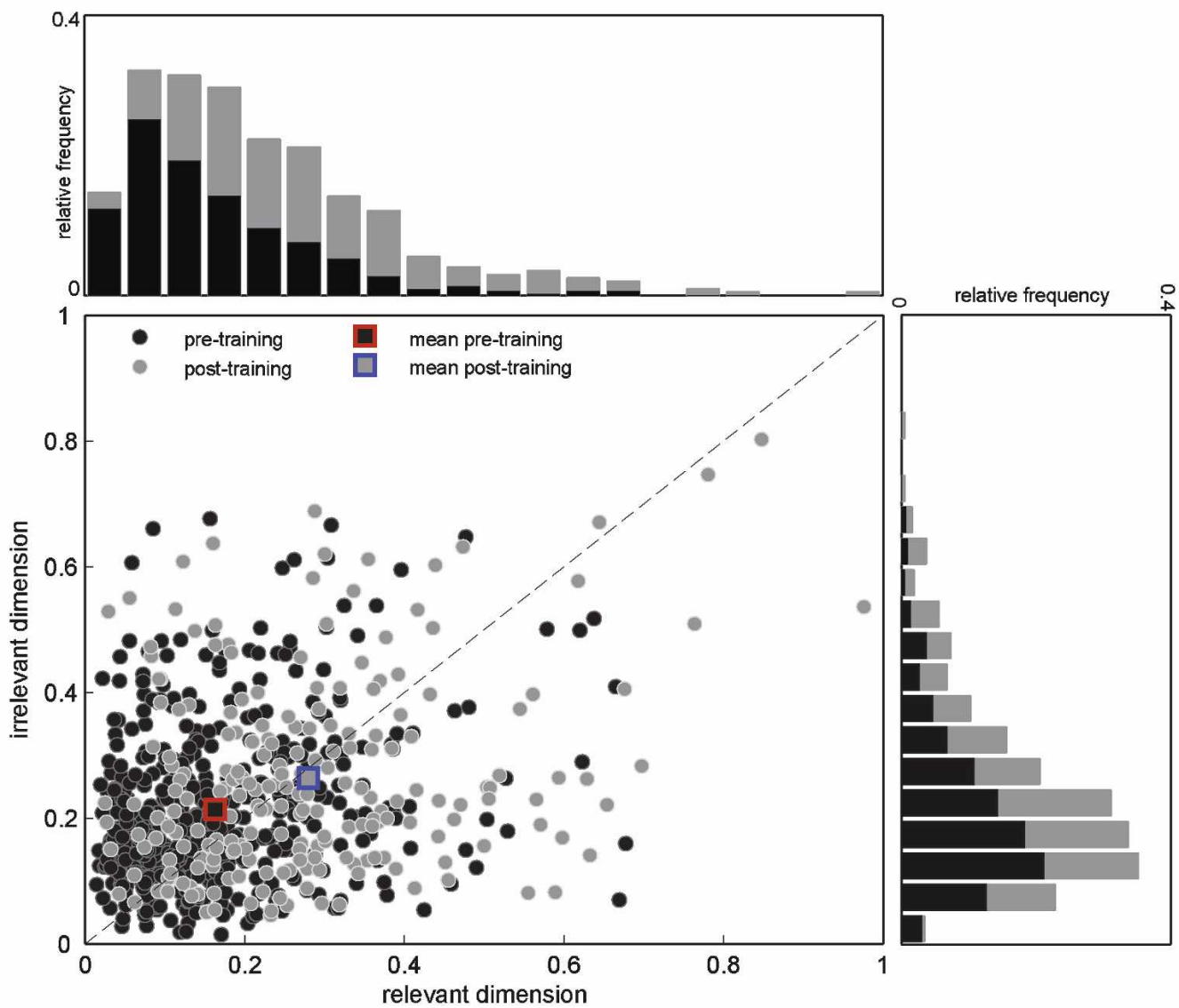

Figure 3. Effect of categorization learning on degree of selectivity. (A) The DOS indices compared before and after categorization training are shown The results for monkey $C$ using curvature as the relevant dimension are shown at left; at right, the results for monkey $A$ using aspect ratio as the relevant dimension are shown. The results before and after training are depicted by dotted and solid lines, respectively (with SEs). (B) The DOS indices compared before and after categorization training for the relevant and irrelevant dimension, pooled across the two monkeys are shown. The results before and after training are depicted by dotted and solid lines, respectively (with SEs). (C) The DOS indices for the irrelevant dimension are plotted against the DOS indices for the relevant dimension, both for the data obtained before (black dots) and after categorization training (gray dots). Means are indicated by the colored symbols. In the marginal histograms, the relative frequencies of the DOS indices for the relevant and irrelevant dimension are shown. The indices obtained before training are depicted by black and gray bars, respectively. Gray dotted rectangles outline the results for the relevant dimension for each monkey.

values for all selective sets in the post-training phase with category dimension (aspect ratio or curvature) as a within-neuron variable and shape set (set 1 to 4 ), center type (type 1 or 2 ), and monkey (monkey $\mathrm{C}$ or A) as between-neurons variables. The interaction between dimension and monkey did not reach significance $\left(F_{(1,394)}=1.80, P>0.17\right)$, indicating that the selectivity for the relevant dimension was not significantly enhanced compared with the irrelevant dimension. This was the case for both center types (DOS index for relevant dimension minus DOS index for irrelevant dimension: center type $1=0.008$; center type $2=-0.034)$. The results for the best-worst selectivity index used by Sigala and Logothetis (2002) were, again, very similar. 
A

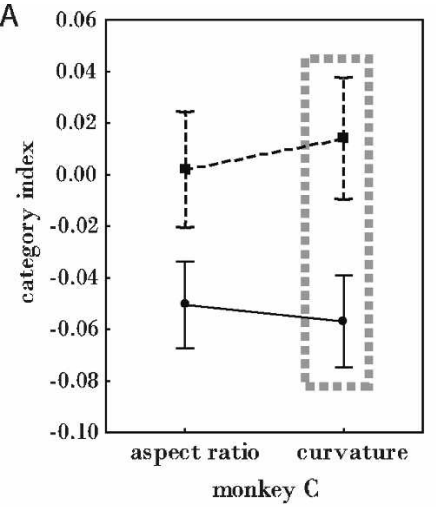

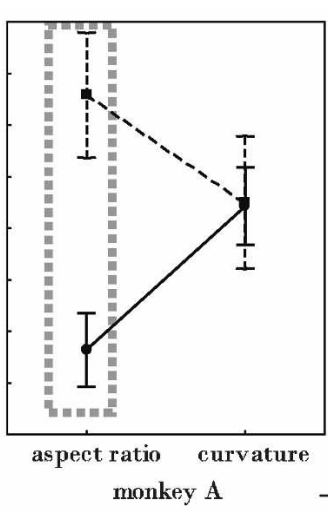

monkey A

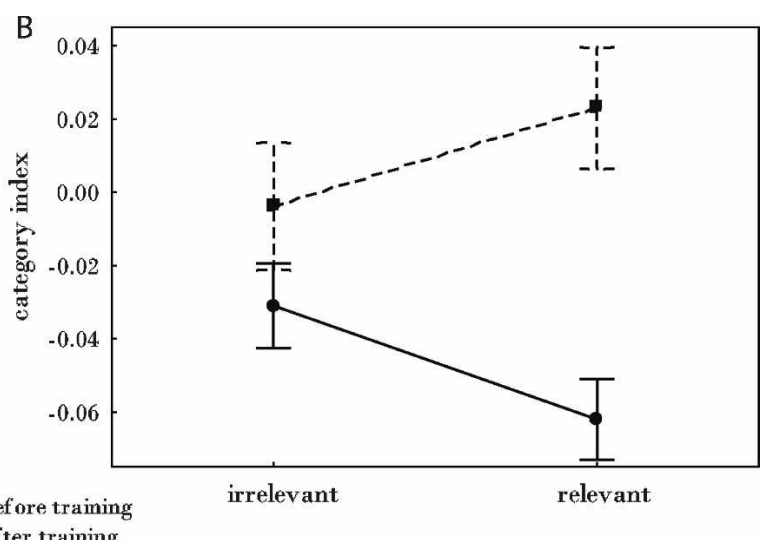

C

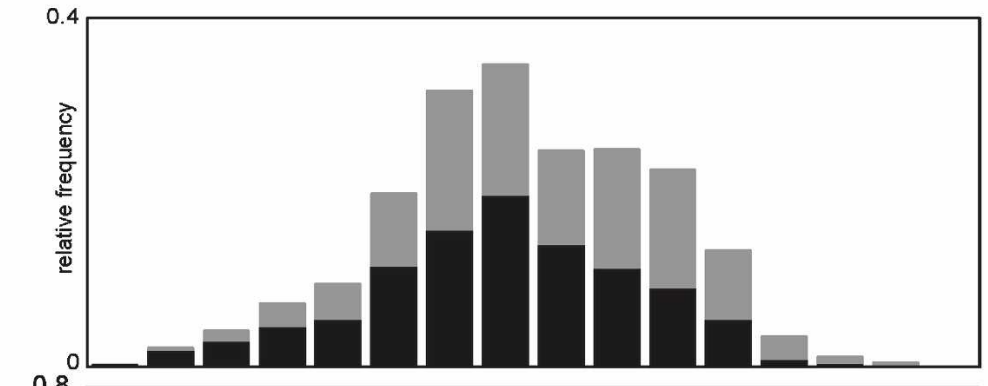



0.8
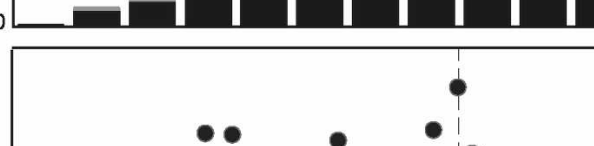

- pre-training
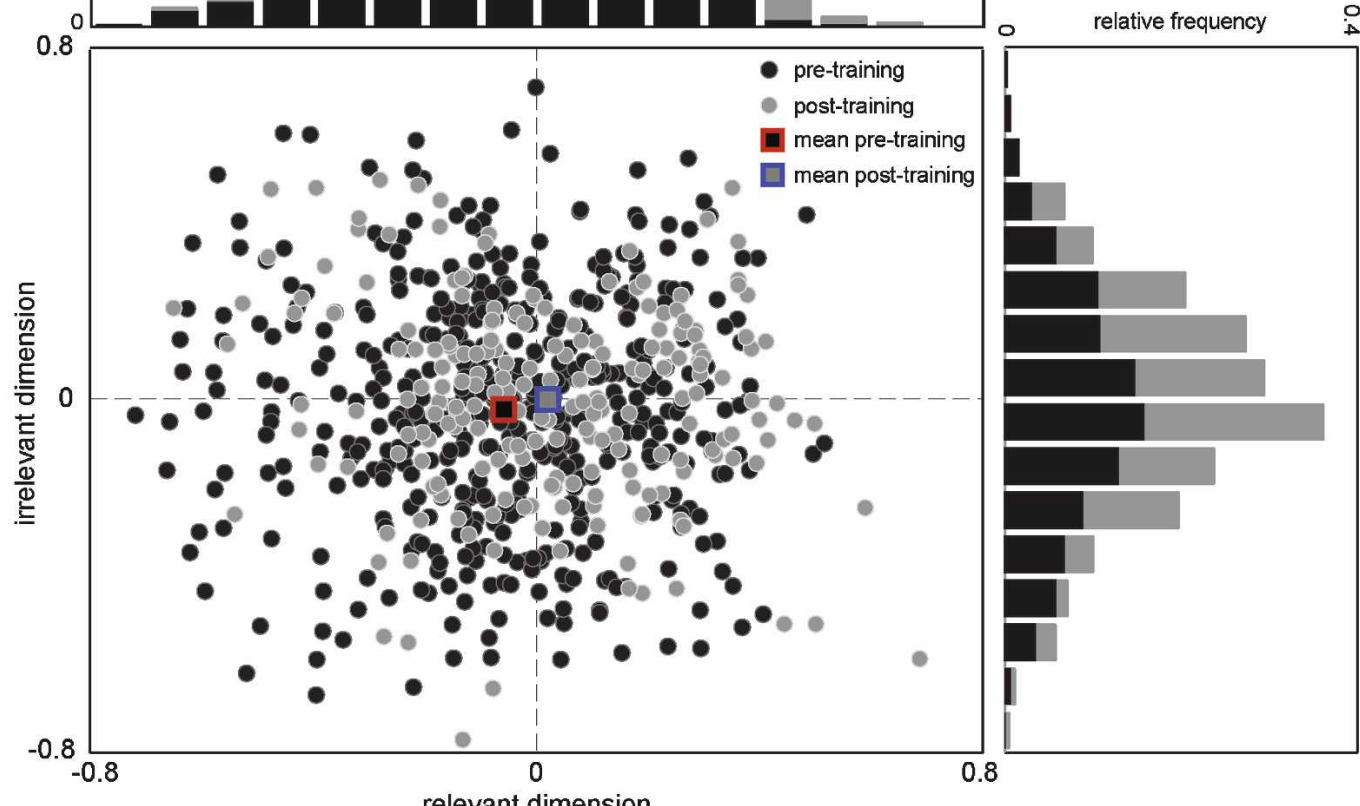

relevant dimension

Figure 4. Effect of category learning on category tuning. (A) Category tuning indices along the curvature and aspect ratio dimensions compared before and after categorization training. The results for the monkey using curvature as the relevant dimension are displayed on the left; on the right, the results for the monkey using aspect ratio as the relevant dimension are shown. The data obtained before and after training are depicted by dotted and solid lines, respectively (with SES). (B) The category tuning indices compared before and after categorization training for the relevant and irrelevant dimension, pooled across the two monkeys, is shown. The data obtained before and after training are depicted by dotted and solid lines, respectively (with SEs). (C) The category tuning indices for the irrelevant dimension are plotted against the category tuning indices for the relevant dimension. Same conventions as in Figure 3.

Strength of category tuning along the relevant vs. irrelevant dimension after training

To examine whether the strength of the category tuning was greater along the relevant dimension than along the irrelevant dimension after training, we used an ANOVA on the category tuning index values for all selective sets in the post-training phase. As between-neurons variables, we included shape set (set 1 to 4 ), center type (type 1 or 2 ), and monkey (monkey C or A).
Category dimension (aspect ratio or curvature) was included as a within-neuron variable. Although the interaction between dimension and monkey did not reach significance $\left(F_{(1,387)}=1.29\right.$, $P>0.25)$, the category tuning along the relevant dimension was greater compared with the irrelevant dimension, which was the case for both center types (category tuning for relevant dimension minus category tuning for irrelevant dimension: center type $1=.027$; center type $2=0.019$ ). A highly similar pattern of non- 
significant results was found when the analysis was applied using the absolute response differences, as Freedman et al. (2003) did, instead of using the $\mathrm{d}^{\prime}$ values.

\section{Discussion}

We examined the effects of categorization learning for shapes upon the selectivity of IT neurons for these shapes, while we controlled for prelearning stimulus selectivity effects versus learned category-related effects by recording the neuronal selectivity before and after categorization learning, as well as by counterbalancing the relevant categorization dimensions across animals.

Unlike previous studies, our design allowed us to simultaneously examine two different possible effects of categorization learning on the tunings of IT neurons. First, IT neurons showed an increased sensitivity for shapes that varied along the trained dimension compared with shapes that varied along the irrelevant dimension. This categorization-relevant selectivity effect was relatively small and could only be demonstrated when comparing pre- and post-training data. Sigala and Logothetis (2002) reported a much stronger effect of categorization training on shape tuning. In the present study, only $55 \%$ of the cells showed a larger selectivity (as quantified by the best-worst selectivity index used by Sigala and Logothetis [2002]) for the relevant compared with the irrelevant dimension after training, which is much smaller than the $\sim 75 \%$ found by Sigala and Logothetis (2002) (quantified after visual inspection of the results in their Fig. 4, A,B). The discrepancy between the size of the effects in the present and the latter study is unlikely to be due to differences in the amount of training. Another reason for the discrepancy between the two studies might be that Sigala and Logothetis had only post-training data and the dimensions actually relevant to the categorization were not counterbalanced across monkeys. Without proper counterbalancing, our results could have been as follows: If curvature had been the relevant dimension for both monkeys, the selectivity for this relevant dimension would have been larger than for the irrelevant aspect ratio dimension for $68 \%$ of the cells when using post-training data or $69 \%$ of the cells when using pretraining data. These proportions are close to those reported by Sigala and Logothetis (2002). However, if aspect ratio had been the relevant dimension for both monkeys, only $32 \%$ (post-training) or 31\% (pretraining data) of the cells would have shown this result pattern. This exercise shows that without proper counterbalancing of the trained dimension, the contribution of a genuine learning effect to the observed selectivity is impossible to assess. Thus, it is possible that if Sigala and Logothetis had counterbalanced the trained dimensions across their subjects, the size of the categorization effect on selectivity would have been much smaller and comparable to that of the present study.

The present results disagree with Op de Beeck et al. (2001), who did not find an effect of categorization rules on IT response tunings. However, training was somewhat less extensive $(\sim 6-8$ wk) than that in the present study and likely more critical, integral, but not separable dimensions (as in the present study and in Sigala and Logothetis [2002]) were manipulated. Perhaps category selective effects on IT tuning occur only for separable dimensions, in line with a psychophysical study of category learning effects (Op de Beeck et al. 2003).

In addition to the dimension-specific change in degree of selectivity, we observed a general increase in selectivity when comparing pre- and post-training selectivity measures. As the procedures of the pre- and post-training recordings differed in several aspects (e.g., fixation vs. categorization, different presentation durations), it is impossible to determine the source of the overall difference in selectivity between the pre- and posttraining phases. De Baene et al. (2007) reported that a RSVP task with a fast presentation rate (100 msec/image) can lead to an underestimation of the degree of stimulus selectivity. This suggests that the overall increase in selectivity with categorization training that was observed in the present study can be at least partially explained by an initial underestimation of the stimulus selectivity caused by the use of a fast presentation-rate RSVP task. However, given previously reported effects of learning on overall shape selectivity (Miyashita et al. 1993; Kobatake et al. 1998; Baker et al. 2002; Freedman et al. 2006), it seems very likely that at least part of the difference in selectivity observed before and after the training is due to a genuine effect of categorization learning on shape selectivity. Note that the different paradigms when searching and testing responsive neurons before and after training cannot explain the dimension-specific changes in selectivity, because the relevant dimension was counterbalanced across animals.

Dimension-specific changes in perceptual sensitivity have been observed after categorization learning in humans using similar (Op de Beeck et al. 2003) or identical shapes (Wagemans et al. 2006) as those used in our study, and in monkeys using other shapes (Sigala et al. 2002). In those behavioral studies, the subjects were performing a task that was different from the trained categorization task when testing their perceptual sensitivity. In our study (as in other single cell categorization studies) (e.g., Vogels 1999b; Op de Beeck et al. 2001; Sigala and Logothetis 2002; Freedman et al. 2003) the animals were performing the categorization task during the post-training recordings, which raises the possibility that the selectivity change is only present when the animals are doing the categorization. Such a taskrelated selectivity change may reflect category dimensionspecific attention, as postulated in exemplar-based models of categorization (e.g., Nosofsky 1986; Kruschke 1992). Note though that the present study would be the first one to show such stimulus dimension-dependent attention effect in the ventral visual stream, since previous studies that specifically examined such attention effects on tuning failed to find it (Mirabella et al. 2007; see Vogels 2007). Although the tuning changes observed in the present study agree with the attentional effect postulated in categorization models, it is possible that the dimension-specific selectivity effect is task independent, and thus might, at least partially, underlie the task-independent changes in behavioral sensitivities that result from categorization learning. To ascertain this, post-training RSVP measures would have been necessary, which were unfortunately not possible in the present study (see Materials and Methods).

Second, IT neurons responded more similarly to shapes belonging to the same category than to shapes belonging to different categories. Since the categorization-related effect is a change in the similarity of the IT responses to shapes that belong to the same versus different categories, and thus differs from a dimension-specific change in shape selectivity (see above), it is difficult to see how this category effect can be explained by an increased attention to the relevant dimension. However, since the two categories were associated with two different behavioral responses, one could argue that the category effect does not reflect a taskindependent change in shape tuning, but instead reflects the upcoming behavioral response. Previous studies that used the same task as we have did not find evidence for such behavioral response-related responses in IT cortex (Op de Beeck et al. 2001; Baker et al. 2002; Koida and Komatsu 2007; also, see Mogami and Tanaka 2006). Given the lack of clear evidence for behavioral response-related effects in these IT studies, it is very unlikely that the category effect that we observed is related to the behavioral response. 
During the categorization training, a small region around the category boundary was spared, which resulted in a slight difference in stimulus statistics for the two monkeys. As a consequence, an unsupervised learning process could have led to a clustering of responses according to the two categories. Although we cannot exclude a possible contribution of this unsupervised learning to the observed category effect, it is very unlikely that it is its sole or main cause. Indeed, the trained shapes near the category boundary were very similar, as is evident from the poor classification performance for these shapes in both animals (about 70\% correct classifications).

Whatever the cause of the category tuning effect, it is numerically rather small. Freedman et al. (2003) reported a similar small effect of categorization learning upon category tuning of IT neurons (after $>6$ mo of training) (D. Freedman, pers. comm.), although, unlike that in our study, their effect could have been due to pretraining stimulus selectivity. Recent human fMRI studies did not find evidence for such category-related tuning in area LOC (believed to be the human homolog of monkey area IT) (Denys et al. 2004) using an adaptation protocol (Jiang et al. 2007) or pattern classification tool (Li et al. 2007). However, effects as small as those observed in the present study may easily be missed with fMRI. Given the modest size of the category-related tuning effect in IT, the question arises as to which area(s) more strongly encode the category membership of a stimulus after learning. Freedman et al. (2003) observed much stronger category tuning effects in prefrontal than in IT neurons when animals performed categorization in the context of a working memory task. Also, several human functional imaging studies of categorization (Vogels et al. 2002; Jiang et al. 2007; Li et al. 2007; for review, see Keri 2003; Ashby and Maddox 2005) reported activation in prefrontal cortex during categorization. A recent study by Freedman and Assad (2006) reported strong categoryrelated information in a working memory task in the intraparietal sulcus. Because motion stimuli were used in this study, it remains unclear whether the parietal areas play a role in categorization tasks in general (thus, this is also when static shapes are used), or only when categorizing motion stimuli (Li et al. 2007). Several functional imaging studies in humans also suggest an involvement of the striatum in categorization (Vogels et al. 2002; Li et al. 2007; for review, see Keri 2003; Ashby and Maddox 2005). As the striatum can integrate the outputs of multiple IT neurons (Cheng et al. 1997), a striatal role in categorization is indeed possible. Note that we cannot exclude that at least part of the effect of category learning on the tuning of IT neurons is caused by feedback from one of the category selective areas.

The present study suggests that shape-categorization learning in adults induces only minor category-related changes in the shape tuning of IT neurons. These changes are such that, at least when post-training responses during categorization are compared with pretraining responses in a fixation task, the representation of the categorization-relevant stimulus features are enhanced, and that the responses of the neurons are somewhat more similar for exemplars that belong to the same, compared with different, categories (Figs. 3, 4, respectively). Our data support a two-stage model of categorization in adults, in which IT neurons are tuned to exemplars, although in a category-biased way, while learned categories become explicitly represented in extra-visual cortical regions that read-out IT. Note that the twostage model proposed here differs from the HMAX model (Riesenhuber and Poggio 1999; Jiang et al. 2007) by allowing a category related, biased object representation in IT, which is not present in HMAX. It is possible that during development, i.e., in animals younger than the ones used in the present study, categorization learning has much stronger category-related effects on the feature representations in the visual cortex, and shapes the category-biased representation as was found by, e.g., Kiani et al. (2007) for animate versus inanimate objects.

\section{Materials and Methods}

\section{Subjects}

Two male rhesus monkeys (Macaca mulatta; Monkeys C and A) served as subjects. Before conducting the experiments, we performed aseptic surgery under isoflurane anesthesia to attach a plastic head-fixation post to the skull and to stereotactically implant a plastic recording chamber. To allow a vertical approach, we positioned the recording chambers dorsal to IT (see Janssen et al. 2000).

All animal care and experimental and surgical procedures followed national and European guidelines and were approved by the K.U. Leuven Ethical Committee for animal experiments.

\section{Stimuli}

Starting with four "archetypical" two-dimensional shapes, we generated four parameterized two-dimensional shape sets by manipulating the dimensions "curvature" and "aspect ratio" (Fig. 1). All archetypical shapes (data not shown in Fig. 1) were chosen so as to display vertical and horizontal symmetry.

Aspect ratio was defined as the ratio between the width and the height of a stimulus. To manipulate this ratio, we modified the width of the archetypical shapes by a transformation that maintained horizontal and vertical symmetry. Curvature was manipulated by modifying the turning angle of the tangents at the lowest and highest point of the vertical axis of the symmetric archetypes (see Foster and Wagemans 1993).

Before carrying out the aspect ratio transformation, we ran a human psychophysical study $(n=6)$, on the basis of which the aspect ratio of the archetypical stimuli were set so as to be perceptually equal to the aspect ratio of a rectangle with vertical sides twice the length of the horizontal sides. We performed a second pilot study with human participants $(n=5)$ to calibrate the aspect ratio and curvature transformations to achieve perceptually equidistant steps for both stimulus dimensions (B. Ons, W. De Baene, and J. Wagemans, in prep.). A third pilot study with human participants $(n=3)$ showed that the introduced shape transformations along the aspect ratio and curvature dimension were fairly perceptual separable, and we were therefore able to combine the two transformations in a linear way to create a twodimensional stimulus set based on the previously defined transformations (B. Ons, W. De Baene, and J. Wagemans, in prep.).

To prevent the possibility that shape categorization might be performed on the basis of shape width instead of aspect ratio, we generated three different shape sizes by a scaling transformation. The mean heights of the large, intermediate, and small sizes were $9.8^{\circ}\left(\min =9.5^{\circ} ; \max =10.1^{\circ}\right), 5.6^{\circ}\left(\min =5.4^{\circ} ; \max =5.8^{\circ}\right)$, and $3.2^{\circ}\left(\min =3.1^{\circ} ; \max =3.3^{\circ}\right)$, respectively. As the turning angle is scale invariant (Foster and Wagemans 1993), this scaling did not affect the curvature of the shapes. Note that it is still possible that the monkeys might have performed the categorization task on the basis of features that vary with the modulated dimensions, rather than using these dimensions themselves. However, as these changes are intertwined with variations in aspect ratio or curvature, we will use the labels "aspect ratio" and "curvature" for the remainder of this study.

All shapes were presented on a gray background on a monitor positioned $60 \mathrm{~cm}$ from the monkeys $(60 \mathrm{~Hz}$ frame rate; $1024 \times 768$ pixels) and were filled with pixel noise. The mean luminance of the shapes and background was equal.

\section{Procedure}

Eye position was monitored by virtue of an infrared eye-tracking system (ISCAN, EC-240A) at a sampling rate of $120 \mathrm{~Hz}$. An electronic fixation window insured that the monkeys maintained their gaze within $0.8^{\circ}$ of a black fixation target $\left(0.17^{\circ}\right.$ diameter $)$ that was presented in the center of the display during the trials.

Standard extracellular recordings were performed with Tungsten microelectrodes that were placed using a guiding tube 
fixed in a Crist grid using previously published techniques (De Baene et al. 2007). Recording positions were estimated by comparing depth readings of the white and gray matter transitions, and that of the skull base, during electrode penetrations, with structural magnetic resonance (MRI) images taken in between the recordings (using a copper-sulfate-filled tube inserted in the grid at one of the recording positions). In monkey $\mathrm{C}$, we recorded from the left hemisphere; in monkey A, we recorded from the right hemisphere. Across animals, the recording positions in both pre- and post-training recording phases (see below) ranged from 15 to $22 \mathrm{~mm}$ anterior to the external auditory meatus and included the lower bank of the superior temporal sulcus (STS), the cortical convexity lateral to the anterior middle temporal sulcus (aMTS), and the lip of the STS (area Tem) (Seltzer and Pandya 1978). The distribution of recording locations in the preand post-training recording phases largely overlapped. Additionally, in the post-training phase, we recorded from the left hemisphere in monkey A as a control. The recording positions in that hemisphere were within the same range as those for the other hemisphere.

\section{Experimental phases}

We planned four experimental phases: a pre-training "baseline" recording using a passive fixation task, a categorization training phase, a post-training recording during categorization, and a post-training recording during passive fixation. However, monkey $\mathrm{C}$ died from a gastrointestinal disorder after the post-training recordings during categorization, precluding post-training measurements using the passive fixation task. Thus, the experimental design included only three phases, which will be described below.

\section{Pretraining recordings}

During the pretraining recordings, we used 16 stimuli of intermediate size of each of the four sets (64 stimuli in total) (Fig. 1). These stimuli were obtained by combining four fixed levels of the curvature parameter with four fixed levels of the aspect ratio parameter. The four fixed curvature levels were combined with each of the four levels of the aspect ratio as shown in Figure 5. The $0 \%, 33 \%, 66 \%$, and $100 \%$ aspect ratios of Figure 5 corresponded to respective horizontal expansions of $1.75,1.32,0.99$, and 0.75 times the archetypical shapes. The four curvature values corresponded to turning angles of the vertical axis of 3.34, 2.23, 1.29 , and 0.52 radians (Fig. 5). The ranges used here were imposed by the intrinsic properties of the shape sets themselves. Higher values on any one of these dimensions would have introduced additional features for the respective stimuli.

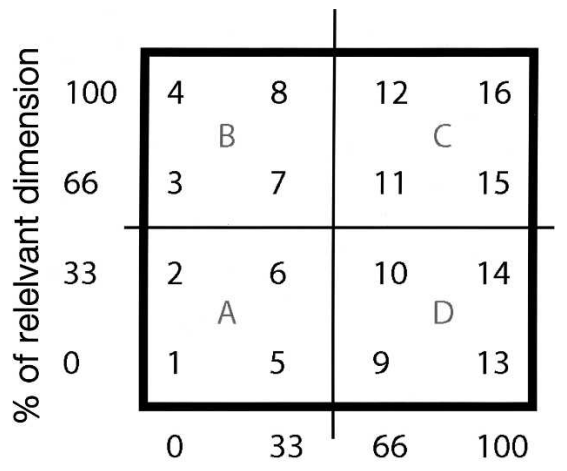

\section{$\%$ of irrelelvant dimension}

Figure 5. Schematic representation of how the shape sets were constructed. The numbers 1 to 16 indicate the different shapes obtained by combining four levels $(0 \%, 33 \%, 66 \%$, and $100 \% ; 0 \%$ and $100 \%$ being the extremes of the range tested on this dimension) of the relevant parameter with the same four levels of the irrelevant parameter. $(A-D)$ The four different quadrants of the configurations from which the position control shapes were chosen. The category boundary is depicted by the horizontal line.
For every shape, a $90^{\circ}$ rotated version was generated, resulting in $128(4 \times 16 \times 2)$ different stimuli. All of these shapes were filled with three different pixel noise patterns, yielding a total of 384 stimuli. The position of the shapes on the screen was determined by the center of the archetypical shape of the respective set. This archetypical shape was centered so that the middle of the shape on the horizontal axis at middle height was at the center of the screen. The curvature and aspect ratio were modulated after this centering. This manner of centering is further referred to as center type 1 .

The 384 stimuli were presented in continuous, rapid, random sequences (no interstimulus interval) at a rate of $100 \mathrm{msec} /$ image (as in De Baene et al. 2007). In this passive fixation task, a trial started after $500 \mathrm{msec}$ of stable fixation and ended when the monkey broke fixation or when every stimulus had been presented once. Monkeys were rewarded with a drop of apple juice at an increasing pace until the end of the trial. This procedure was used to search for responsive neurons. If the onlinegenerated peristimulus time histogram (PSTH; averaged across trials and all stimuli) of the responses indicated that the cell did not respond to any of the stimuli, we abandoned this cell and searched for another. Spikes from responsive cells were sampled for about 75 trials.

\section{Categorization learning}

In a second phase, the monkeys were trained to group the stimuli into two categories, with curvature and aspect ratio as the relevant dimensions for monkeys $\mathrm{C}$ and A, respectively. Each category was associated with a saccade direction: shapes with parametric values $<50 \%$ on the relevant dimension (below horizontal line, Fig. 5) were associated with a leftward saccade, shapes with parametric values $>50 \%$ (above horizontal line, Fig. 5) with a rightward saccade. In this categorization task, a trial started after $500 \mathrm{msec}$ of stable fixation. A stimulus was presented for 300 msec and after another 200 msec of fixation, two black target spots $\left(0.71^{\circ}\right.$ diameter) appeared to the left and right of the fixation point, at $9^{\circ}$ eccentricity as a response cue. Saccades in the correct direction and within 2000 msec after the onset of the target spots were rewarded with a drop of apple juice. The fixation point and target spots were turned off as the eye trace entered a target window. Trials in which the animal interrupted fixation before target spot onset or failed to saccade to one of the two target spots were counted as aborts, and were not included when computing categorization performance. In contrast to the stimuli in the first recording phase, which were presented at a fixed intermediate size, all shapes in the categorization learning phase were presented at three different sizes (see above). Each shape was presented at a random position within a $3.6^{\circ}$ square region centered on the fixation point and was filled with one of three different pixel noise patterns, which were refreshed daily.

The monkeys first learned to categorize the shapes of set 1 (Fig. 1). Initially, only eight stimuli per size were presented, i.e., four of each extreme level $(0 \%$ and $100 \%)$ of the irrelevant dimension (i.e., shapes 1-4 and 13-16; shapes numbered as in Fig. $5)$. We increased the number of shapes on a daily basis by adding shapes 8 and 9 on day 2 , shapes 7 and 10 on day 3, shapes 5 and 12 on day 4 , and shapes 6 and 11 on day 5 .

For each remaining day of the training phase, we generated 16 novel shapes for each size, eight for each category, with random values along the two dimensions. These values were randomly picked from within the limits of the original shape configuration (Fig. 1) but excluding a small range (having an extent of $\sim 1 / 6$ of the total tested range) symmetrical around the category boundary of the relevant dimension. The category boundary for curvature was defined as the parametric value at the $50 \%$ level, referring to a turning angle of 1.74 radians. The category boundary for aspect ratio was defined as the parametric value, generating shapes with a physical aspect ratio of 0.54 . The reason for this will be discussed below.

After the fifth day of training, we tested the transfer to the other three sets on three consecutive days. For each of these sets, 16 transfer stimuli were generated for each of the three sizes by combining the four fixed levels $(0 \%, 33 \%, 66 \%$, and $100 \%)$ of the 
curvature parameter with the four fixed levels of the aspect ratio parameter (see above). These $48(3 \times 16)$ transfer stimuli within a set were randomly intermixed with 16 randomly generated stimuli per size of set 1 (each presented nine times; three times per pixel noise pattern), and were presented twice, making up $10 \%$ of all trials presented on a given day. These transfer stimuli were always followed by reward, irrespective of the monkey's response (Vogels 1999a). These transfer tests showed that monkey $\mathrm{C}$ was able to generalize the learned category boundary to novel sets, while monkey A had difficulty in doing this generalization: Monkey $\mathrm{C}$ achieved an average performance of $70 \%$ over the three novel sets and performed significantly better than chance for all three sets (tested with a Binomial test; Mean $=62.5 \%, P<0.05$ for set 2 ; Mean $=71 \%, P<0.001$ for set 3 ; Mean $=77 \%, P<0.001$ for set 4 ). Monkey A attained $61 \%$ correct, averaged across the three novel sets, and performed significantly above chance level only for set 3 (tested with a Binomial test; Mean $=60.5 \%, P>0.05$ for set 2 ; Mean $=65.5 \%, P<0.01$ for set 3; Mean $=56 \%, P>0.05$ for set 4 ). It is difficult to know whether the difference in the degree of transfer to novel sets shown by two animals simply reflects a distinction between individual subjects or is instead related to the different trained dimensions.

After this transfer test, we presented stimuli from the four sets randomly intermixed. From this point on, the 16 shapes with fixed values (per size) of sets 2,3 , and 4 were no longer used for the remainder of the training phase (as was already the case for set 1). Instead, every day we randomly picked 16 new stimuli (eight for each category) per size and per set with values from within the limits of the tested configuration. Again, these values could not lie within a small range symmetrical around the category boundary of the relevant dimension (see above). This range of values was identical for all sets for the curvature dimension. This was because shapes from different sets that had equal parametric curvature values indeed had equal curvature. As the archetypical shapes were calibrated to have perceptually equal aspect ratios, the shapes of different sets differed slightly in their physical aspect ratios. To induce category boundaries that were at an equivalent physical aspect ratio level for any given set, for each set we adapted the range around the boundary from which no values could be picked. The 16 shapes that were used in the post-training recording phase (see further) were assigned to the same category across the four sets.

Because monkey $\mathrm{C}$, who performed the categorization task with curvature as the relevant dimension, made very few errors, the range around the category boundary was halved (to $1 / 12$ of the total tested range) for this monkey after the fourth day of performing to criterion level (see below).

Once the monkeys (monkey C: $16 \mathrm{~d}$; monkey A: $42 \mathrm{~d}$ ) had learned to categorize the shapes to criterion performance $(90 \%$ correct responses averaged across all stimuli of all sets and sizes), the training continued for another 37 or $38 \mathrm{~d}$ (for monkey $\mathrm{C}$ and monkey A, respectively).

\section{Post-training recordings}

In a third phase, we recorded from IT cortex while the monkeys were performing the categorization task (see above). We searched for responsive neurons with 16 randomly generated stimuli per size for each of the four sets (using the same randomization as during the training phase; see above), which were presented with three different pixel noise patterns at a random position within a square region of $3.6^{\circ}$ centered at the fixation point. Every day we generated new stimuli and renewed the pixel noise patterns. We visualized the responses of the cells in peristimulus time histograms (PSTHs) averaged across trials and shapes. We had a PSTH for each combination of the four sets and three sizes, resulting in 12 different PSTHs. Based on a visual inspection of these PSTHs, we selected the sets for which a neuron was judged to be responsive for the subsequent main test. Note that this selection of responsive neurons and stimulus sets was unbiased with respect to the tuning for the curvature or aspect ratio dimensions.

To maximize the data collected on a given recording day, we selected a maximum of two shape sets for the subsequent test. If the neuron responded to shapes from more than two sets, we randomly chose two sets from these possibilities and started the subsequent test with the randomly intermixed presentation of the shapes of these two sets. The remaining sets were introduced only in a subsequent test once we had sampled enough trials (i.e., minimally 10 trials per stimulus) for the first two chosen sets. The recording of a cell was aborted when all shapes of all selected sets had been presented about 10 times, when it became impossible to isolate the cell's responses from the other neuronal activity, or when the monkey simply stopped working.

In this main test, we presented, per selected set, the 16 shapes with fixed parametric levels (as in Figs. 1, 5) filled with one pixel noise pattern (which was refreshed every day) at an intermediate size at a fixed position.

Given the inherent difficulty of centering stimuli that vary in curvature and the fact that the animals were trained to categorize the stimuli at randomly chosen positions, we also tested neurons with a second type of centering (in addition to the one used in the pretraining recording phase [center type 1]). This allowed us to generalize the (absence of) effects of categorization on neural selectivity for different positioning of the stimuli (see Results). For this center type 2 , the middle of the vertical, curved medial axis was centered on the screen. This center type 2 was used in about half of all cells recorded in the post-training phase and for all cells recorded in the left (control) hemisphere in monkey A. Note that all analyses that compare pre- and post-training responses utilized only data obtained with the same centering (center type 1). The difference between the two types of centering was such that the aspect ratio or curvature transformations were first applied before the shape was centered in center type 2, while the shape was centered before application of the stimulus transformations in center type 1.

In addition to the 16 fixed shapes per selected set, eight catch stimuli per set were added for behavioral control only: four size controls and four position controls. The size control stimuli included two large and two small shapes, with random parametric values lying within the limits of the tested configuration and with the constraint that each category was chosen with equal frequency. From each quadrant of the 16-shape configuration (A-D; Fig. 5), one shape of intermediate size was presented at a control position. The maximum difference between the position control shapes and their corresponding reference shapes was $1.5^{\circ}$.

\section{Data analysis and tests}

In both pre- and post-training recordings, the response of the neuron was defined as the mean number of spikes in a 70-200 msec analysis window relative to stimulus onset. The selection of the analysis window was based on an analysis of the monkeys' eye movements during the categorization task in the posttraining phase (Fig. 6). After about $150 \mathrm{msec}$, one of the monkeys made small, preparatory saccades toward the correct-response side, but still within the fixation window (Fig. 6). These small saccades can, in principle, alter the visual input to the retina, which could in turn affect the neuronal responses in IT. To avoid any possible effect that this altered retinal input might have on the responses of the IT cells (which could occur after about 220 msec, given that the minimum latency of IT neurons is $\sim 70 \mathrm{msec}$ ) (see Vogels 1999b), we chose an analysis window up to $200 \mathrm{msec}$ after stimulus onset.

The response of a neuron recorded in the pretraining phase was measured using the same procedure as in De Baene et al. (2007), excluding the first three stimuli as well as the last stimulus of a trial sequence. However, a response window of 70-200 msec post-stimulus onset was used (instead of 50-200 msec in De Baene et al. 2007). All analyses in the pretraining phase were performed on neurons showing shape selectivity. For every cell recorded in the pretraining phase, we tested the shape selectivity for each stimulus set using a permutation test to assess the statistical significance of the observed variance within a given shape set in the mean neuronal responses to stimuli. For each shape set, the distribution of the variances expected by chance was determined by calculating new variances of the data after permuting 

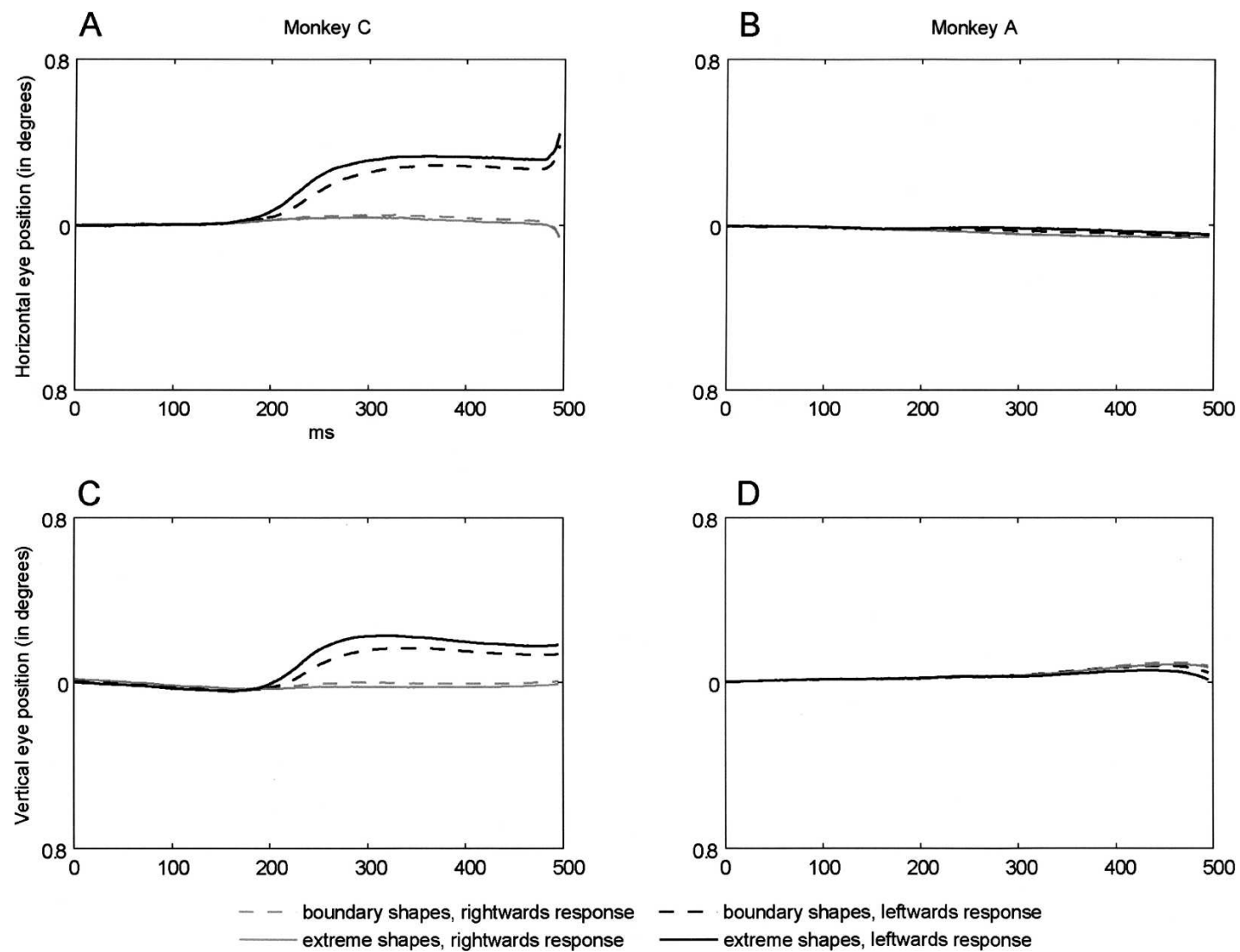

Figure 6. Eye positions averaged over trials for monkey $C(A, C)$ and monkey $\mathrm{A}(B, D)$ during the categorization task in the post-training recording phase. At top $(A, B)$, the mean horizontal eye positions (in visual degrees) are displayed. At bottom $(C, D)$, the mean vertical eye positions are presented. On the $X$-axis, the stimulus presentation time relative to stimulus onset is plotted; on the $Y$-axis, the eye position within the fixation window is plotted. Values $0.8,0$, and $-0.8^{\circ}$ (from top to bottom) represent the left/top $(A, B / C, D)$ boundary, the center and the right/bottom $(A, B / C, D)$ boundary of the average fixation window, respectively. Eye positions for shapes associated with a rightward or leftward saccade are plotted in gray and black, respectively. Eye positions for shapes close to the category boundary (i.e., with a parametric value of $33 \%$ or $66 \%$ ) are displayed in dotted lines. Solid lines are used for shapes with extreme parametric values (i.e., $0 \%$ or $100 \%$, and thus not close to the category boundary).

the order of the stimuli within each trial while maintaining the actual spike counts. We generated a distribution of 1000 permuted variances, representing the distribution of variances that would have been expected to occur by a chance association between stimulus and neuronal firing. A neuron was considered to be shape selective within a shape set if the observed variance for responses within that shape set was larger than the 95th percentile of the values in its own permuted variance distribution $(P<0.05,1$-tailed $)$.

The responsiveness of cells in the post-training phase was assessed per set by a split-plot-design analysis of variance (ANOVA) (Kirk 1995) comparing baseline activity, measured in a -130-0 msec time window relative to stimulus onset, with stimulus-driven activity measured in a 70-200 msec time window, relative to stimulus onset. All analyses in the post-training phase were performed on the sets, for which at least a main effect of this response variable $(P<0.05)$ was found. A significant interaction $(P<0.05)$ between this response variable and shape indicated that the cell was selective within this set. Comparisons between pre- and post-training phase were performed only for these shape selective neurons.

In both pre- and post-training recording phases, we calculated several indices for both the relevant and irrelevant dimension (thus, for both the learned and unlearned categories, respectively) per tested set. Only trials with correct responses were included in these analyses. As a measure of the selectivity for the relevant versus the irrelevant dimension, we computed per set the depth of selectivity (DOS) (Rainer and Miller 2000) for each dimension separately. This measure of the degree of selectivity of a neuron to a given stimulus set was defined as:

$$
\left[n-\left(\sum_{i=1}^{n} R_{i} / R_{\max }\right)\right] /(n-1)
$$

where $n=$ number of parametric values of the relevant/irrelevant dimension (i.c. 4: $0 \%, 33 \%, 66 \%$, and $100 \%$ ); $R_{\mathrm{i}}=$ mean firing rate (averaged across the four values of the irrelevant/relevant dimension, respectively) to the $i$ th parametric value of the relevant/irrelevant dimension; and $R_{\max }=\max \left\{R_{\mathrm{i}}\right\}$. The DOS could vary between 0 (when the neuron responded equally for all parametric values) and 1 (when there was a response for only one parametric value). We used the DOS index rather than a bestworst index (Sigala and Logothetis 2002), since the DOS index takes into account responses to all, rather than only two, of the parametric values. As for the best-worst index used by Sigala and Logothetis (2002), the DOS indices were computed on the marginal means of the parametric values of a dimension, i.e., by averaging across the mean responses for the four values of the other dimension (e.g., response for curvature $0 \%$ represents the average of the mean responses for the shapes having the $0 \%$, $33 \%, 66 \%$, and $100 \%$ aspect ratio values of that curvature value).

To compare differences in responses to shapes belonging to the same versus different categories, we computed, for each neuron and set, the absolute differences in average response strength to all possible pairs of adjacent stimuli (separately along the relevant and irrelevant dimension of the shape configuration). These response differences were then divided by the square root of the mean of the variances in the response strength to these stimuli. These $\mathrm{d}^{\prime}$ values take into account the difference in mean spike counts as well as the variance of the response over trials. 
The differences for pairs of stimuli that belonged to the same category were defined as the within-category differences (WCDs); between-category differences (BCDs) were calculated between adjacent stimuli belonging to two different categories. The average parametric distance between stimuli was identical for BCDs and WCDs. An index of the strength of category tuning was computed for each neuron by dividing the difference between the averages of the BCDs and WCDs by the sum of these averages, giving values ranging from -1 to 1 , where positive values indicate larger response differences between categories compared with within a category, and negative values indicate the opposite. This category tuning index is identical to the one used by Freedman et al. (2003) in their analysis of category tuning, except that we computed the category tuning index using $\mathrm{d}^{\prime}$ values instead of raw response differences.

\section{Acknowledgments}

The technical assistance of P. Kayenbergh, G. Meulemans, M. De Paep, and S. Verstraeten is gratefully acknowledged. We thank R. Peeters for his help in taking structural magnetic resonance images from our monkeys. This research was supported by Geneeskundige Stichting Koningin Elizabeth ELT-B3972, GOA/ 2005/18, IUAP P6/29, Detection and Identification of Rare Audiovisual Cues FP6-IST 027787, EF/05/014, and IDO/02/004.

\section{References}

Afraz, S.R., Kiani, R., and Esteky, H. 2006. Microstimulation of inferotemporal cortex influences face categorization. Nature 442: 692-695.

Ashby, F.G. and Maddox, W.T. 2005. Human category learning. Annu. Rev. Psychol. 56: 149-178.

Baker, C.I., Behrmann, M., and Olson, C.R. 2002. Impact of learning on representation of parts and wholes in monkey inferotemporal cortex. Nat. Neurosci. 5: 1210-1215.

Cheng, K., Saleem, K.S., and Tanaka, K. 1997. Organization of corticostriatal and corticoamygdalar projections arising from the anterior inferotemporal area TE of the macaque monkey: A Phaseolus vulgaris leucoagglutinin study. J. Neurosci. 17: 7902-7925.

Dean, P. 1976. Effects of inferotemporal lesions on the behavior of monkeys. Psychol. Bull. 83: 41-71.

De Baene, W., Premereur, E., and Vogels, R. 2007. Properties of shape tuning of macaque inferior temporal neurons examined using rapid serial visual presentation. J. Neurophysiol. 97: 2900-2916.

Denys, K., Vanduffel, W., Fize, D., Nelissen, K., Peuskens, H., Van Essen, D., and Orban, G.A. 2004. The processing of visual shape in the cerebral cortex of human and nonhuman primates: A functional magnetic resonance imaging study. J. Neurosci. 24: 2551-2565.

Foster, D.H. and Wagemans, J. 1993. Viewpoint-invariant Weber fractions and standard contour-curvature discrimination. Biol. Cybern. 70: 29-36.

Freedman, D.J. and Assad, J.A. 2006. Experience-dependent representation of visual categories in parietal cortex. Nature 443: $85-88$.

Freedman, D.J., Riesenhuber, M., Poggio, T., and Miller, E.K. 2003. A comparison of primate prefrontal and inferior temporal cortices during visual categorization. J. Neurosci. 23: 5235-5246.

Freedman, D.J., Riesenhuber, M., Poggio, T., and Miller, E.K. 2006. Experience-dependent sharpening of visual shape selectivity in inferior temporal cortex. Cereb. Cortex 16: 1631-1644.

Garner, W.R. 1974. The processing of information and structure. Erlbaum, Potomac, MD

Goldstone, R.L. 1994. Influences of categorization on perceptual discrimination. J. Exp. Psychol. Gen. 123: 178-200.

Goldstone, R.L., Lippa, Y., and Shiffrin, R.M. 2001. Altering object representations through category learning. Cognition 78: 27-43.

Gross, C.G., Bender, D.B., and Rocha-Miranda, C.E. 1969. Visual receptive fields of neurons in inferotemporal cortex of the monkey. Science 166: 1303-1306.

Janssen, P., Vogels, R., and Orban, G.A. 2000. Selectivity for 3D shape that reveals distinct areas within macaque inferior temporal cortex. Science 288: 2054-2056.

Jiang, X., Bradley, E., Rini, R.A., Zeffiro, T., VanMeter, J., and Riesenhuber, M. 2007. Categorization training results in shape- and category-selective human neural plasticity. Neuron 53: 891-903.
Kayaert, G., Biederman, I., and Vogels, R. 2003. Shape tuning in macaque inferior temporal cortex. J. Neurosci. 23: 3016-3027.

Kayaert, G., Biederman, I., Op de Beeck, H., and Vogels, R. 2005. Tuning for shape dimensions in macaque inferior temporal cortex. Eur. J. Neurosci. 22: 212-224.

Keri, S. 2003. The cognitive neuroscience of category learning. Brain Res. Brain Res. Rev. 43: 85-109.

Kiani, R., Esteky, H., Mirpour, K., and Tanaka, K. 2007. Object category structure in response patterns of neuronal population in monkey inferior temporal cortex. J. Neurophysiol. 97: 4296-4309.

Kirk, R.E. 1995. Experimental design procedures for the behavioral sciences. Brooks/Cole, Pacific Grove, CA.

Kobatake, E., Wang, G., and Tanaka, K. 1998. Effects of shape-discrimination training on the selectivity of inferotemporal cells in adult monkeys. J. Neurophysiol. 80: 324-330.

Koida, K. and Komatsu, H. 2007. Effects of task demands on the responses of color-selective neurons in the inferior temporal cortex. Nat. Neurosci. 10: $108-116$.

Kruschke, J.K. 1992. ALCOVE: An exemplar-based connectionist model of category learning. Psychol. Rev. 99: 22-44.

Li, S., Ostwald, D., Giese, M., and Kourtzi, Z. 2007. Flexible coding for categorical decisions in the human brain. J. Neurosci. 27: 12321-12330.

Livingston, K.R., Andrews, J.K., and Harnad, S. 1998. Categorical perception effects induced by category learning. J. Exp. Psychol. Learn. Mem. Cogn. 24: 732-753.

Logothetis, N.K. and Sheinberg, D.L. 1996. Visual object recognition. Annu. Rev. Neurosci. 19: 577-621.

Mirabella, G., Bertini, G., Samengo, I., Kilavik, B.E., Frilli, D., Della Libera, C., and Chelazzi, L. 2007. Neurons in area V4 of the macaque translate attended visual features into behaviorally relevant categories. Neuron 54: 303-318.

Miyashita, Y., Date, A., and Okuno, H. 1993. Configurational encoding of complex visual forms by single neurons of monkey temporal cortex. Neuropsychologia 31: 1119-1131.

Mogami, T. and Tanaka, K. 2006. Reward association affects neuronal responses to visual stimuli in macaque TE and Perirhinal cortices. J. Neurosci. 26: 6761-6770.

Nosofsky, R.M. 1986. Attention, similarity, and the identification categorization relationship. J. Exp. Psychol. Gen. 115: 39-57.

Op de Beeck, H., Wagemans, J., and Vogels, R. 2001. Inferotemporal neurons represent low-dimensional configurations of parameterized shapes. Nat. Neurosci. 4: 1244-1252.

Op de Beeck, H., Wagemans, J., and Vogels, R. 2003. The effect of category learning on the representation of shape: Dimensions can be biased but not differentiated. J. Exp. Psychol. Gen. 132: 491-511.

Rainer, G. and Miller, E.K. 2000. Effects of visual experience on the representation of objects in the prefrontal cortex. Neuron 27: 179-189.

Riesenhuber, M. and Poggio, T. 1999. Hierarchical models of object recognition in cortex. Nat. Neurosci. 2: 1019-1025.

Seltzer, B. and Pandya, D.N. 1978. Afferent cortical connections and architectonics of the superior temporal sulcus and surrounding cortex in the rhesus monkey. Brain Res. 149: 1-24.

Sigala, N. and Logothetis, N.K. 2002. Visual categorization shapes feature selectivity in the primate temporal cortex. Nature 415: $318-320$.

Sigala, N., Gabbiani, F., and Logothetis, N.K. 2002. Visual categorization and object representation in monkeys and humans. J. Cogn. Neurosci. 14: $187-198$.

Tanaka, K. 1996. Inferotemporal cortex and object vision. Annu. Rev. Neurosci. 19: 109-139.

Thomas, E., Van Hulle, M.M., and Vogels, R. 2001. Encoding of categories by noncategory-specific neurons in the inferior temporal cortex. J. Cogn. Neurosci. 13: 190-200.

Vogels, R. 1999a. Categorization of complex visual images by rhesus monkeys. Part 1: Behavioural study. Eur. J. Neurosci. 11: 1223-1238.

Vogels, R. 1999b. Categorization of complex visual images by rhesus monkeys. Part 2: Single-cell study. Eur. J. Neurosci. 11: 1239-1255.

Vogels, R. 2007. Representation of response categories in visual cortex. Neuron 54: 181-183.

Vogels, R., Sary, G., Dupont, P., and Orban, G.A. 2002. Human brain regions involved in visual categorization. Neuroimage 16: 401-414.

Wagemans, J., Ons, B., Gillebert, C.R., and Op de Beeck, H. 2006. Effects of categorization learning on perceived shape similarity in humans. Soc. Neurosci. Abstr. 438: 22.

Received April 18, 2008; accepted in revised form June 19, 2008. 


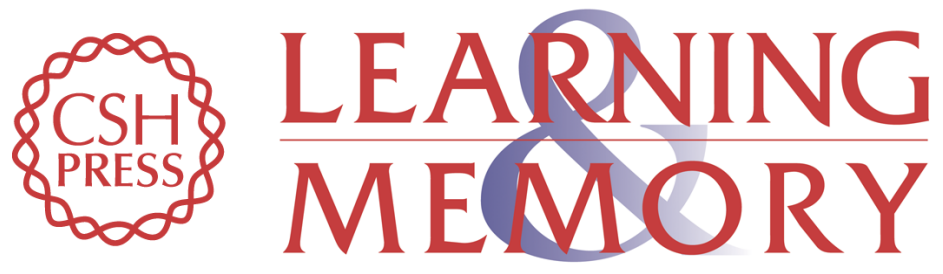

\section{Effects of category learning on the stimulus selectivity of macaque inferior temporal neurons}

Wouter De Baene, Bart Ons, Johan Wagemans, et al.

Learn. Mem. 2008, 15:

Access the most recent version at doi:10.1101//m.1040508

References This article cites 44 articles, 8 of which can be accessed free at:

http://learnmem.cshlp.org/content/15/9/717.full.html\#ref-list-1

License

Email Alerting Receive free email alerts when new articles cite this article - sign up in the box at the Service top right corner of the article or click here. 